\title{
The interplay between excitons and trions in a monolayer of $\mathrm{MoSe}_{2}$
}

Cite as: Appl. Phys. Lett. 112, 031107 (2018); https://doi.org/10.1063/1.5019177

Submitted: 19 May 2017. Accepted: 11 December 2017 . Published Online: 17 January 2018

N. Lundt, E. Cherotchenko, O. Iff, X. Fan, Y. Shen, P. Bigenwald, A. V. Kavokin (D), S. Höfling, and C. Schneider
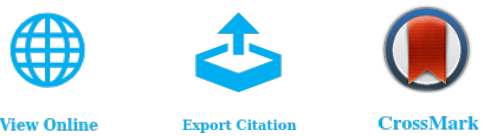

\section{ARTICLES YOU MAY BE INTERESTED IN}

Broadband nonlinear optical response of monolayer $\mathrm{MoSe}_{2}$ under ultrafast excitation

Applied Physics Letters 112, 031108 (2018); https://doi.org/10.1063/1.5010060

Giant excitation induced bandgap renormalization in TMDC monolayers

Applied Physics Letters 112, 061104 (2018); https://doi.org/10.1063/1.5017069

Topological optical isolator based on polariton graphene

Applied Physics Letters 112, 031106 (2018); https://doi.org/10.1063/1.5018902

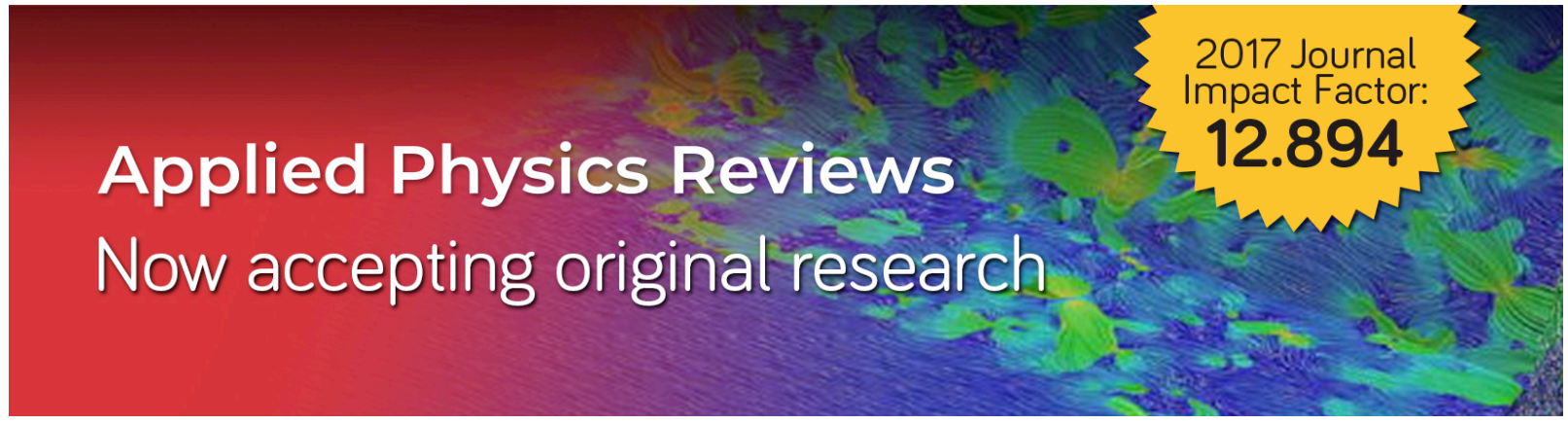




\title{
The interplay between excitons and trions in a monolayer of $\mathrm{MoSe}_{\mathbf{2}}$
}

\author{
N. Lundt, ${ }^{1}$ E. Cherotchenko, ${ }^{2}$ O. Iff, ${ }^{1}$ X. Fan, ${ }^{3}$ Y. Shen, ${ }^{3}$ P. Bigenwald, ${ }^{4}$ A. V. Kavokin, ${ }^{2,5}$ \\ S. Höfling, ${ }^{1,6}$ and C. Schneider ${ }^{1}$ \\ ${ }^{1}$ Technische Physik and Wilhelm Conrad Röntgen Research Center for Complex Material Systems, \\ Physikalisches Institut, Universität Würzburg, Am Hubland, D-97074 Würzburg, Germany \\ ${ }^{2}$ Physics and Astronomy School, University of Southampton, Highfield, Southampton SO171BJ, \\ United Kingdom \\ ${ }^{3}$ School for Engineering of Matter, Transport, and Energy, Arizona State University, Tempe, Arizona 85287, USA \\ ${ }^{4}$ Institut Pascal, PHOTON-N2, Clermont Universit, Blaise Pascal University, Centre National de la Recherche \\ Scientifique, 24 Avenue des Landais, 63177 Aubire Cedex, France \\ ${ }^{5}$ SPIN-CNR, Viale del Politecnico 1, I-00133 Rome, Italy \\ ${ }^{6}$ SUPA, School of Physics and Astronomy, University of St Andrews, St Andrews KY16 9SS, United Kingdom
}

(Received 19 May 2017; accepted 11 December 2017; published online 17 January 2018)

\begin{abstract}
The luminescence and absorption properties of transition metal dichalcogenide monolayers are widely determined by neutral and charged excitonic complexes. Here, we focus on the impact of a free carrier reservoir on the optical properties of excitonic and trionic complexes in a $\mathrm{MoSe}_{2}$ monolayer at cryogenic temperatures. By applying photodoping via a non-resonant pump laser, the electron density can be controlled in our sample, which is directly reflected in the contribution of excitons and trions to the luminescence signal. We find significant shifts of both the exciton and trion energies in the presence of an induced electron gas both in power- and in time evolution (on the second to minute scale) in our photoluminescence spectra. In particular, in the presence of the photo-doped carrier reservoir, we observe that the splitting between excitons and trions can be enhanced by up to $4 \mathrm{meV}$. This behaviour is phenomenologically explained by an interplay between an increased screening of excitons via electrons in our system and a modification of the Fermi level. We introduce a simple but still quantitative treatment of these effects within a variational approach that takes into account both screening and phase space filling effects. Published by AIP Publishing. https://doi.org/10.1063/1.5019177
\end{abstract}

Monolayers of transition metal dichalcogenides (TMDCs) are a very suitable system to study the physics of excitons, as well as fundamental light matter coupling in solid state systems, since they feature very distinct, well isolated excitonic and trionic resonances even at room temperature. $^{1,2}$ This is a consequence of the interplay between quantum confinement, reduced dielectric screening, and heavy effective electron and hole masses in TMDC monolayers. ${ }^{3}$ While devices such as light emitting diodes, ${ }^{4,5}$ solar cells, ${ }^{6}$ ultra-fast photodetectors, ${ }^{7}$ and single- as well as photon-pair emitters ${ }^{8-13}$ have been demonstrated already, the full potential of TMDCs for fundamental studies of excitonlight coupling is yet to be exploited. This involves the integration of monolayers into microcavities to observe the formation of exciton-polariton resonances, ${ }^{14-16}$ which are very promising towards the implementation of valley polarized macroscopic quantum states. ${ }^{17}$ In this field, the behavior of Coulomb-correlated carrier complexes in the presence of a carrier gas is of particular interest potentially featuring interesting effects such as the formation of polarons and polaritons at Fermi-edge singularities ${ }^{18}$ and even exciton-mediated superconductivity. ${ }^{19-21}$

In order to investigate and understand these effects, a profound understanding of the interaction between excitons, trions, and free carriers is absolutely essential. This includes subtle energy shifts and renormalization of excitonic and trionic binding energies due to the presence of macroscopic exciton reservoirs as well as free carriers, which has been observed in other material systems. ${ }^{22-25}$
$\mathrm{MoSe}_{2}$ monolayers are particularly promising for studies of such effects, since their emission spectra feature a strong, spectrally well separated trion signal. On most substrates, no defect related, potentially disturbing features are observed, as compared to $\mathrm{WSe}_{2}, \mathrm{WS}_{2}$, and $\mathrm{MoS}_{2}$ monolayers for example. This clear spectrum makes $\mathrm{MoSe}_{2}$ monolayers well suitable for studying the effect of excess carriers on these Coulomb-correlated complexes.

Experimentally, the influence of a free carrier gas on the excitonic response in monolayers of $\mathrm{MoS}_{2}$ and $\mathrm{WS}_{2}{ }^{3,26,27}$ has been investigated in absorption measurements close to the Mott transition in gated devices. Similar results have recently been confirmed in photoluminescence measurements on $\mathrm{WS}_{2}{ }^{28}$ and $\mathrm{MoSe}_{2}$ monolayers using photodoping to create free carriers. ${ }^{29}$ However, the results on $\mathrm{MoSe}_{2}$ only indicated slightest changes in trion dissociation energy on the sub-meV scale, without providing a quantitative theory to gain more insight into the underlying effects. Here, we enhance the amount of photodoping on $\mathrm{MoSe}_{2}$ monolayers at cryogenic temperatures using higher excitation powers and longer optical illumination times, which is a realistic configuration for polariton experiments in the non-linear regime. In this scenario, we were able to observe changes in the trion dissociation energy up to $4 \mathrm{meV}$, which reflects that both the exciton and the trion binding energies in $\mathrm{MoSe}_{2}$ sensibly depend on the excess carrier density in the monolayer. While previous all-optical investigations were rather focused on the origin of the photo-doping process, ${ }^{29}$ we provide a model for the interaction between free carriers, excitons, and trions. This model is based on the 
original variational approach that takes into account both screening and phase space filling effects. This model is in good quantitative agreement with our experimental findings.

Monolayers of $\mathrm{MoSe}_{2}$ were deposited onto $285 \mathrm{~nm}$ thermal oxide on $\mathrm{Si}$ wafers via conventional exfoliation from bulk crystals. The $\mathrm{SiO}_{2}$ thickness was chosen to be $285 \mathrm{~nm}$ to improve the monolayer contrast in our optical microscope. First, the exfoliated monolayers were characterized using Raman and photoluminescence spectroscopy to check their optical quality. Photoluminescence spectra were recorded at $5 \mathrm{~K}$, via exciting the monolayer non-resonantly with a frequency doubled Nd:YAG laser at $532 \mathrm{~nm}$. The excitation laser is utilized to create excitons and trions in our monolayer as well as to activate additional carriers in the heterostructure.

Figure 1(a) depicts a series of photoluminescence spectra of such a $\mathrm{MoSe}_{2}$ monolayer under non-resonant photo-excitation at $532 \mathrm{~nm}$. In this experiment, the intensity of the nonresonant pump laser, which was focused to a spot with a diameter of approx. $3 \mu \mathrm{m}$, was successively increased from $500 \mu \mathrm{W}$ to $10 \mathrm{~mW}$ to study the evolution of the two dominant emission features. At each power, the illumination was held for $10 \mathrm{~min}$. We note that we do not see any other dominant emission features over a wide spectral range (hundreds of $\mathrm{meV})$, and the agreement of the energetic positions $(1.662 \mathrm{eV}$ vs. $1.631 \mathrm{eV}$ ) of the two signals with previous works at modest pump power justifies their assignment as the neutral exciton and the negatively charged trion. ${ }^{30,31}$ Furthermore, residual ndoping is commonly observed in TMDC monolayers, which makes the appearance of a negatively charged trion more likely than a positively charged complex. Along with the monotonous increase in the peak intensity, the emission features are subject to a broadening of their emission linewidth, as depicted in Fig. 1(b). The continuous increase in the emission linewidth of both the exciton and trion signals can be related to a combination of effects. One could be an increased dephasing of the carrier complexes interacting with a successively increasing carrier gas in the background. In addition, the pump laser induces heat in our system, which results in an increasing excitation of phonons, which couple to our excitons and cause a broadening of the emission linewidth. ${ }^{32}$

We will now comparatively monitor the powerdependent emission features of the $\mathrm{X}$ and $\mathrm{X}$ - complexes in our sample. Thus, we re-plot the series of emission spectra shown in Fig. 1(a) by normalizing each spectrum to the excitonic signal, as shown in Fig. 2(a). This procedure reveals the increasing dominance of the trion feature towards higher excitation powers and indicates that the non-resonant pump laser a

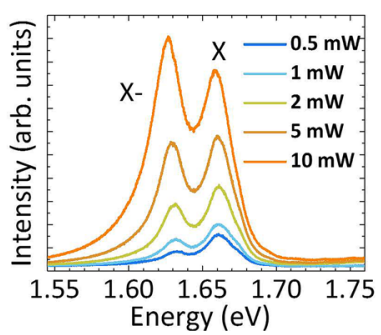

b

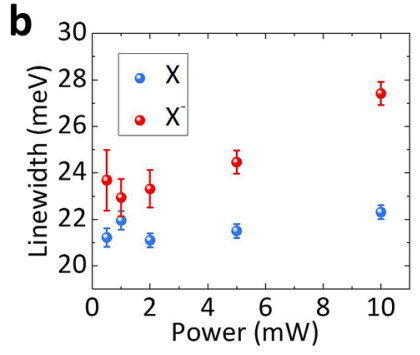

FIG. 1. (a) Power series of the $\mathrm{MoSe}_{2}$ monolayer at $5 \mathrm{~K}$ under $532 \mathrm{~nm}$ excitation (spectra were taken at the beginning of the 10-min-illumination at each power). (b) Corresponding linewidths of excitonic and trionic resonances. induces additional electrons in our sample, which enhance the formation of trionic complexes. Most likely, this activation stems from optical ionization of carrier trap centers in the substrate or the substrate-monolayer interface, which is followed by a transfer of free charge carriers into the monolayer. ${ }^{29}$

A more controlled method to tune the electron density without significantly modifying the excitonic density in our monolayer is shown in Fig. 2(b): Conveniently, the photoexcitation process of additional free carriers takes place on a very slow timescale (seconds to minutes). Thus, the fraction of excitons versus trions can be tuned simply by adjusting the illumination time of the monolayer. In addition, we plot the ratio of the integrated intensities of the trion and exciton emission peaks as functions of the exposure time in Fig. 2(b), for various illumination times and excitation powers. Here, it becomes clear that the exciton vs. trion intensity ratio also changes over time at a fixed excitation power, which takes place on the minute scale.

At smallest excitation powers, we are in a regime where the exciton signal is still dominating the spectrum. However, even with constant pump power, the $\mathrm{X}$-/X fraction is already monotonously increasing with time, exhibiting a saturation behaviour. This indicates a self-limiting activation process, typical for the successive activation of a finite number of defect states. An increase in the pump power then leads to a further increase in this ratio, which serves as a proportional measure for the number of electrons per exciton in our system. ${ }^{29}$ Within the pump power range of $0.5 \mathrm{~mW}$ to $10 \mathrm{~mW}$ (power measured in front of our microscope objective) and an exposure time up to $60 \mathrm{~min}$, we manage to tune the $\mathrm{X}-/ \mathrm{X}$ fraction between a number as low as 0.3 up to 2.2. This gives us a convenient basis to study electron-hole correlation energies in the presence of a free electron gas. We note that while charging the sample, the exciton intensity shows sub-linear behavior. In contrast, in the regime of a stable $\mathrm{X} / \mathrm{X}$ - ratio, both the exciton and the trion exhibit a linear dependency on the pump power (not shown), thus indicating the strong influence of a changing carrier density. Furthermore, these observations are fully repeatable after heating up the sample to room temperature and exposing it to ambient air. However, after a high power exposure, the $\mathrm{X}-\mathrm{X}$ ratio remains high even under low excitation power as observed in Ref. 29. Therefore, the sample was exactly illuminated for $10 \mathrm{~min}$ and we strictly measured in the consecutively increasing power steps. We thus investigate the emission energies of both optical resonances as a function of both the pump power and the exposure time, which are directly correlated with the excess electron density.
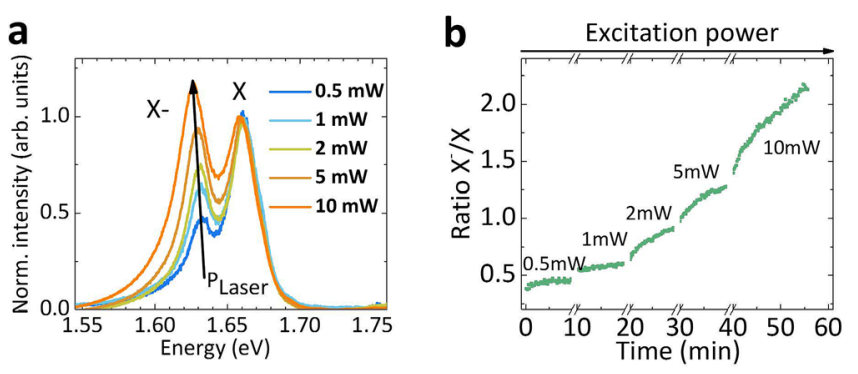

FIG. 2. (a) Power series of PL spectra normalized to the excitonic resonance. (b) Ratio of integrated peak intensities as a function of time and excitation power. 
We extract the peak energies of the $\mathrm{X}$ and $\mathrm{X}$ - resonances by fitting the spectra to a double Gaussian envelope. Due to the almost background-free spectra, the energies of both resonances can be accurately extracted (better than $100 \mu \mathrm{eV}$ ). The evolution of the energies of both the exciton and the trion as functions of the exposure time is subject to a stepwise increase in the pump power shown in Fig. 3(a). As the excitation power is increased, both the $\mathrm{X}$ and $\mathrm{X}$ - signals experience a similar red-shift, resulting from the powerinduced renormalization of the bandgap of the monolayer. The contribution of the microscopic processes of this renormalization is not fully determined (dielectric screening vs. sample heating); however, it is of minor importance in our study, since the energy scale is approximately two orders of magnitude smaller than the correlation energies of our excitonic complexes. A second effect, which can be directly observed in Fig. 3(a), is the shift of both the X and X-signals as the pump laser intensity is kept at the same level. Here, the behaviour of both resonances is fundamentally different: as the pump intensity is kept constant and charges successively accumulate in the monolayer, the $\mathrm{X}$ feature is subject to a continuous blueshift in energy, whereas the $\mathrm{X}$ - feature is redshifted by a similar magnitude (hundreds of $\mu \mathrm{eV}$ up to $\mathrm{meV})$. The blue-shift of the excitons in the presence of an accumulating electron gas is a strong signature of a renormalisation of the exciton binding energy. In the presence of additional screening, Coulomb coupling between electrons and holes is reduced, ${ }^{33-36}$ and the excitonic emission energy eventually approaches the free carrier bandgap, which is known to be subject to renormalization effects itself as the free carrier density is increased. ${ }^{37}$ In our experiment, from the chosen excitation powers of $0.5-10 \mathrm{~mW}$, we estimate carrier densities on the order of $6.6 \times 10^{9} / \mathrm{cm}^{2}$ to $1.3 \times 10^{11} /$ $\mathrm{cm}^{2}$. This estimate is based on the assumption of an effective excitation relaxation time of approx. $10 \mathrm{ps,} \mathrm{which} \mathrm{is} \mathrm{known}$ to vary between experiments, ${ }^{38}$ and a diameter of our laser spot of approx. $3 \mu \mathrm{m}$. a

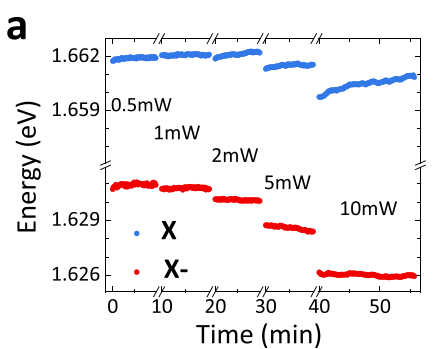

b

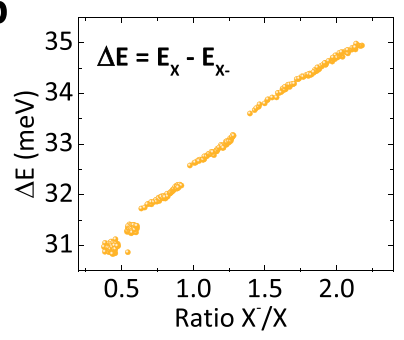

$c$

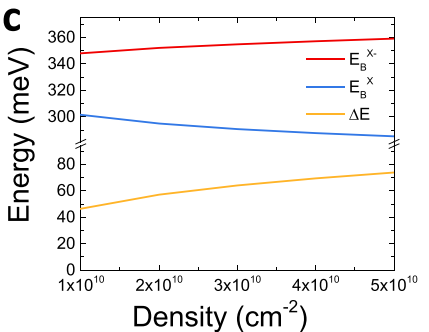

FIG. 3. (a) Evolution of exciton and trion energies with illumination time and excitation power. (b) Trion dissociation energy as a function of $\mathrm{X}-/ \mathrm{X}$ ratio. (c) Exciton and trion binding energies (blue and yellow lines, respectively) and the difference between spectral positions of $\mathrm{X}^{-}$with respect to the single particle band-gap in the effective-exciton approach (red line).
Due to the pump powers, yielding exciton densities well below the Mott density in $\mathrm{MoSe}_{2}$ monolayers (around $10^{13}-10^{14}$ carriers $/ \mathrm{cm}^{2}$ ), the exciton energy is not expected to approach the free particle bandgap.

The redshift of the energy of the trion, on the other hand, is a strong indication that the trion binding energy is enhanced by the electron reservoir, which over-compensates the screening induced blueshift of the exciton. In order to directly correlate the trion dissociation energy with the approximate number of excess carriers per exciton, we plot the energy $\left(E_{X}-E_{X_{-}}\right)$as a function of the $\mathrm{X}-/ \mathrm{X}$ ratio in Fig. 3(b).

We find that the trion dissociation energy in our system is a linear function of the intensity ratio between $\mathrm{X}$ - and $\mathrm{X}$ and thus of the number of electrons in our monolayer. It is worth noting that the overall value of the trion dissociation energy increases from 31 to approx. $35 \mathrm{meV}$ in our experiment. The proportionality between the $\mathrm{X}-/ \mathrm{X}$ ratio and the number of electrons cannot necessarily be taken for granted but should hold true in this regime of modest carrier density. The fact that this linear behavior is furthermore present throughout the stepwise increases in the pump power additionally justifies our interpretation and evidences that the interaction of excitons and trions with free carriers is the main reason of these energy shifts. We also like to note that the electronic bandgap is expected to be subject to renormalization effects. However, this renormalization would act on both the exciton and trion energies in the same way and should not affect the trion dissociation energy.

A qualitative explanation of the increase in the trion binding energy in quantum wells was given in Ref. 39 in relation to experiments on the absorption of light in a CdTe QW, containing a two-dimensional electron gas. The separation between trion and exciton peaks turned out to be linear in $n_{e}: E_{x}-E_{t r}=E_{b}^{t r}+c E_{F}$, with $c \approx 1$. Here, $E_{b}^{t r}$ is the trion binding energy and $E_{F}$ is the electron Fermi energy calculated from the bottom of the conduction band. An exciton is considered as an ionized trion. The energy that is needed to remove one electron from the trion is equal to $E_{b}^{t r}$ at $n_{e} \rightarrow 0$ and $E_{b}^{t r}+E_{F}$ at finite electron densities, due to the fact that one electron should be moved up in energy to the Fermi level while all lower conduction band states are occupied. Thus, this effect stems from the combined effect of the Pauli exclusion principle and many-body interactions. Investigations carried out in Refs. 27 and 40 confirm this linear dependence of the trion binding energy in TMDC monolayers with small differences in estimations of coefficient $c$. Here, we assume that the trion is negatively charged due to the residual n-type doping commonly observed in TMDC monolayers. The model $^{39}$ fails to describe the behaviour of exciton-trion energy splitting in the limit of high concentrations of free electrons, where it predicts unphysical negative values. In order to reproduce the experimental data taken in a wide range of free electron densities, one needs to go beyond the oversimplified model ${ }^{39}$ and calculate separately the exciton and trion binding energies, see, e.g., Ref. 40. Here, we exploit the "effective exciton" model ${ }^{41}$ and use a variational approach to the calculation of exciton and trion states which has an advantage of simplicity but captures the behaviour of the system at both low and high electronic densities. 
The Schrodinger equation for the wave-function of the electron-hole relative motion in the plane of the layer $\Psi(\rho)$ in cylindrical coordinates reads

$$
\left[\frac{-\hbar^{2}}{2 \mu} \frac{1}{\rho} \frac{\partial}{\partial \rho}\left(\rho \frac{\partial}{\partial \rho}\right)-\int_{0}^{\infty} J_{0}(k \rho) V_{C} k d k-E_{e x}\right] \Psi(\rho)=0,
$$

where $\mu=\frac{m_{e} m_{h}}{m_{e}+m_{h}}$ is the reduced mass of electron-hole relative motion, $J_{0}(k \rho)$ is the zeroth order Bessel function, and $V_{C}$ is the screened Coulomb potential.

Here, we describe the free carriers as a degenerate Fermi gas at zero temperature. We account for the exclusion effect associated with the phase space filling. All electronic states below the Fermi level are assumed to be occupied, which is why they cannot contribute to the exciton state. Taking into account this exclusion effect, the trial function of the exciton can be written in the form ${ }^{42}$

$$
\begin{aligned}
\Psi(\rho) & =\int_{0}^{\infty} J_{0}(k \rho) f(k) k d k, \\
f(k) & =\frac{B}{\left(A+k^{2}\right)^{3 / 2}} \Theta\left(k-k_{F}\right),
\end{aligned}
$$

where $\Theta\left(k-k_{F}\right)$ is the Heaviside function, $k_{F}=\sqrt{2 \pi n_{2 D}}, n_{2 D}$ is the density of free carriers, and $A$ is a variational parameter, inversely proportional to the squared exciton Bohr radius. $B$ can be found from the normalization condition

$$
\int_{0}^{\infty} \Psi(\rho)^{2} 2 \pi \rho d \rho=1
$$

In the limiting case of $k_{F}=0$, the introduced wave function reduces to the hydrogen-like wave-function

$$
\Psi(\rho)=\sqrt{\frac{2}{\pi}} \frac{1}{a} \exp \left(-\frac{\rho}{a}\right),
$$

where $a$ is the exciton Bohr radius.

Varying the parameter $A$, the exciton binding energy can be found as the minimum value of $E_{e x}$, where

$$
\begin{aligned}
E_{e x}= & \int_{0}^{\infty} \Psi(\rho)\left[\frac{-\hbar^{2}}{2 \mu} \frac{1}{\rho} \frac{\partial}{\partial \rho}\left(\rho \frac{\partial}{\partial \rho}\right)-\int_{0}^{\infty} J_{0}(k \rho) V_{C} k d k\right] \\
& \times \Psi(\rho) 2 \pi \rho d \rho .
\end{aligned}
$$

$$
V_{C}=\frac{e^{2} \arctan (\tilde{k} / k)}{\epsilon k\left(1+(\kappa / k)\left(1-\Theta\left(k-2 k_{F}\right) \sqrt{1-\left(2 k_{F} / k\right)^{2}}\right)\right.} .
$$

Here, $\epsilon$ is the mean dielectric constant that takes into account the substrate, $\kappa=\frac{2 g_{\nu} m_{e} e^{2}}{\epsilon \hbar^{2}}$ is the screening constant, $g_{\nu}$ is the valley degeneracy factor, and $\tilde{k}=2 \pi / 0.75 L$, with $L$ being the thickness of a layer. The electron-density dependent potential (5) is suitable for the description of the dependence of the exciton binding energy on the free carrier concentrations. Its advantages over the frequently used Yukawa potential are addressed in Ref. 44.

For the trion case, we simplify the problem and assume that the two electrons (we consider the $\mathrm{X}^{-}$case without the loss of generality) are in the singlet state so that they are characterized by orthogonal spin functions and identical spatial wave functions. The trion binding energy may be found as the solution of the Coulomb problem with a hole of charge $+e$ and mass $m_{h}$ and an electron pair of charge $-2 e$ and mass $2 m_{e}$. In this case, the trion wave function can be expressed as a sum of two parts, corresponding to the electrons composed by the states that lie below and above the Fermi level, respectively, as follows:

$$
\begin{aligned}
& \Psi(\rho)=\int_{0}^{\infty} J_{0}(k \rho) f_{t r}(k) k d k, \\
& f_{t r}(k)=\frac{B}{\left(A+k^{2}\right)^{3 / 2}} \Theta\left(k-k_{F}\right)+\frac{C}{\left(D+k^{2}\right)^{3 / 2}} \Theta\left(k_{F}-k\right),
\end{aligned}
$$

where $A$ and $D$ are the variational parameters and $B$ and $C$ can be found from normalization conditions. Here, we account for the exclusion principle for both electrons: the photoexcited electron can only be formed by free states, while the resident electron can only be taken from the states below the Fermi level. The good estimate for the trion binding energy is found in a similar manner to that in (4) by minimization over both variational parameters $A$ and $D$.

The described method was used to estimate the exciton and trion binding energies in a $\mathrm{MoSe}_{2}$ monolayer. We have used the following parameters: $\mu_{e x}=0.31 m_{0}{ }^{45}$ and $\epsilon=5 .{ }^{46}$ In the presence of free carriers, this model yields the exciton binding energy of about $E=350 \mathrm{meV}$. The results for exciton and trion binding energies at different electron densities calculated with the described model are shown in Fig. 3(c). The binding energies in the presence of free carriers calculated with this approach are somewhat lower than the experimental values for $\mathrm{MoSe}_{2}{ }^{47}$ This might be improved by taking the screening more accurately, e.g., using the potential, defined in Refs. 48 and 49 instead of modified Yukawa potential, which includes only density dependence. Another possible way of analysis is using the many-body approach as it is performed in Ref. 50. However, the approach developed here has an advantage of the utmost simplicity that allows tracing the main dependencies quasi-analytically. It correctly describes the experimental data in what concerns the dependence of the energy splitting between exciton and trion resonances on the free carrier density in the chosen experimental range. It can be seen that at low densities ( 3 orders of magnitude below the Mott density), the energy difference increases linearly with electron density.

We have investigated the Coulomb correlation energies of photo-excited excitons and trions in monolayers of $\mathrm{MoSe}_{2}$ at cryogenic temperatures. By taking advantage of an inherent photoexcitation of additional charge carriers in our structure, we were able to tune the ratio between exciton and trion peak intensities by a factor of 7 . We observe a continuous screening of the exciton on the meV scale, and more importantly, a simultaneous increase of the trion binding energy up to $4 \mathrm{meV}$ as the electron density is increased. These experimental findings are reproduced with the variational calculation that accounts for the screening and phase space filling effects for excitons and trions. Our considerations clearly demonstrate that the Trion dissociation energy 
is a subtle quantity which strongly depends on both the dielectric environment and the experimental conditions. We anticipate that the capability to tune the Exciton-Trion splitting over an energy range by pure photodoping, in conjunction with the highest quality monolayer samples,${ }^{29}$ will certainly shed some light on phenomena associated with the phonon-mediated coupling between excitons and trions in $\mathrm{MoSe}_{2}{ }^{51}$ Further, we believe that our findings are of fundamental interest in the emerging research field devoted to Fermi-Bose mixtures, in particular, targeting to explore interactions of bosonic excitations in the presence of a Fermi-sea. Understanding subtle power-depending signatures of the primary resonances in monolayers of TMDCs will help harnessing electron-exciton coupling to induce superconductivity mediated by excitons.

We acknowledge financial support by the ERC (Grant Unlimit-2D) and the State of Bavaria.

We acknowledge S. Tongay for his assistance in sample preparation. A.K. acknowledges the support from SaintPetersburg State University within the research grant 11.34.2.2012.

\section{${ }^{1}$ L. Cao, MRS Bull. 40, 592-599 (2015).}

${ }^{2}$ K. F. Mak and J. Shan, Nat. Photonics 10, 216-226 (2016).

${ }^{3}$ A. Chernikov, T. C. Berkelbach, H. M. Hill, A. Rigosi, Y. Li, O. B. Aslan, D. R. Reichman, M. S. Hybertsen, and T. F. Heinz, Phys. Rev. Lett. 113, 076802 (2014).

${ }^{4}$ J. S. Ross, P. Klement, A. M. Jones, N. J. Ghimire, J. Yan, M. G. T. Taniguchi, K. Watanabe, K. Kitamura, W. Yao, D. H. Cobden, and X. Xu, Nat. Nanotechnol. 9, 268-272 (2014).

${ }^{5}$ B. W. H. Baugher, H. O. H. Churchill, Y. Yang, and P. Jarillo-Herrero, Nat. Nanotechnol. 9, 262-267 (2014).

${ }^{6}$ A. Pospischil, M. M. Furchi, and T. Mueller, Nat. Nanotechnol. 9, 257-261 (2014).

${ }^{7}$ O. Lopez-Sanchez, D. Lembke, M. Kayci, A. Radenovic, and A. Kis, Nat. Nanotechnol. 8, 497-501 (2013).

${ }^{8}$ Y.-M. He, G. Clark, J. R. Schaibley, Y. He, M.-C. Chen, Y.-J. Wei, X. Ding, Q. Zhang, W. Yao, X. Xu, C.-Y. Lu, and J.-W. Pan, Nat. Nanotechnol. 10, 497-502 (2015).

${ }^{9}$ A. Srivastava, M. Sidler, A. V. Allain, D. S. Lembke, A. Kis, and A. Imamolu, Nat. Nanotechnol. 10, 491-496 (2015).

${ }^{10}$ C. Chakraborty, L. Kinnischtzke, K. M. Goodfellow, R. Beams, and A. N. Vamivakas, Nat. Nanotechnol. 10, 507-511 (2015).

${ }^{11}$ M. Koperski, K. Nogajewski, A. Arora, V. Cherkez, P. Mallet, J.-Y. Veuillen, J. Marcus, P. Kossacki, and M. Potemski, Nat. Nanotechnol. 10, 503-506 (2015).

${ }^{12}$ P. Tonndorf, R. Schmidt, R. Schneider, J. Kern, M. Buscema, G. A. Steele, A. Castellanos-Gomez, H. S. J. van der Zant, S. Michaelis de Vasconcellos, and R. Bratschitsch, Optica 2, 347-352 (2015).

${ }^{13}$ Y.-M. He, O. Iff, N. Lundt, V. Baumann, M. Davanco, K. Srinivasan, S. Höfling, and C. Schneider, Nat. Commun. 7, 13409 (2016).

${ }^{14}$ N. Lundt, S. Klembt, E. Cherotchenko, S. Betzold, O. Iff, A. V. Nalitov, M. Klaas, C. P. Dietrich, A. V. Kavokin, S. Höfling et al., Nat. Commun. 7, 13328 (2016).

${ }^{15}$ S. Dufferwiel, S. Schwarz, F. Withers, A. Trichet, F. Li, M. Sich, O. Del Pozo-Zamudio, C. Clark, A. Nalitov, D. Solnyshkov et al., Nat. Commun. 6, 8579 (2015).

${ }^{16}$ L. C. Flatten, Z. He, D. M. Coles, A. A. Trichet, A. W. Powell, R. A. Taylor, J. H. Warner, and J. M. Smith, Sci. Rep. 6, 33134 (2016).
${ }^{17}$ N. Lundt, A. Maryński, E. Cherotchenko, A. Pant, X. Fan, S. Tongay, G. Sek, A. Kavokin, S. Höfling, and C. Schneider, 2D Mater. 4, 015006 (2016).

${ }^{18}$ M. Sidler et al., Nat. Phys. 13, 255-261 (2017).

${ }^{19}$ O. Cotlet et al., Phys. Rev. B 93, 054510 (2016).

${ }^{20}$ F. Laussy et al., Phys. Rev. Lett. 104, 106402 (2010).

${ }^{21}$ E. D. Cherotchenko et al., Superlattices Microstruct. 90, 170-175 (2016).

${ }^{22}$ G. V. Astakhov, D. R. Yakovlev, V. P. Kochereshko, W. Ossau, J. Nürnberger, W. Faschinger, and G. Landwehr, Phys. Rev. B 60, R8485-R8488 (1999).

${ }^{23}$ G. V. Astakhov, V. P. Kochereshko, D. R. Yakovlev, W. Ossau, J. Nürnberger, W. Faschinger, G. Landwehr, T. Wojtowicz, G. Karczewski, and J. Kossut, Phys. Rev. B 65, 115310 (2002).

${ }^{24}$ G. V. Astakhov, D. R. Yakovlev, V. P. Kochereshko, W. Ossau, W. Faschinger, J. Puls, F. Henneberger, S. A. Crooker, Q. McCulloch, D. Wolverson, N. A. Gippius, and A. Waag, Phys. Rev. B 65, 165335 (2002).

${ }^{25}$ K. Kheng, R. T. Cox, M. Y. d'Aubigné, F. Bassani, K. Saminadayar, and S. Tatarenko, Phys. Rev. Lett. 71, 1752-1755 (1993).

${ }^{26}$ A. Chernikov, A. M. van der Zande, H. M. Hill, A. F. Rigosi, A. Velauthapillai, J. Hone, and T. F. Heinz, Phys. Rev. Lett. 115, 126802 (2015).

${ }^{27}$ K. F. Mak, K. He, C. Lee, G. H. Lee, J. Hone, T. F. Heinz, and J. Shan, Nat. Mater. 12, 207-211 (2013).

${ }^{28}$ A. Mitioglu, P. Plochocka, J. Jadczak, W. Escoffier, G. Rikken, L. Kulyuk, and D. Maude, Phys. Rev. B 88, 245403 (2013).

${ }^{29}$ F. Cadiz, C. Robert, G. Wang, W. Kong, X. Fan, M. Blei, D. Lagarde, M. Gay, M. Manca, T. Taniguchi, K. Watanabe, T. Amand, X. Marie, P. Renucci, S. Tongay, and B. Urbaszek, 2D Mater. 3, 045008 (2016).

${ }^{30}$ J. S. Ross, S. Wu, H. Yu, N. J. Ghimire, A. M. Jones, G. Aivazian, J. Yan, D. G. Mandrus, D. Xiao, W. Yao et al., Nat. Commun. 4, 1474 (2013).

${ }^{31}$ D. MacNeill, C. Heikes, K. F. Mak, Z. Anderson, A. Kormányos, V. Zólyomi, J. Park, and D. C. Ralph, Phys. Rev. Lett. 114, 037401 (2015).

${ }^{32}$ G. Moody, C. Kavir Dass, K. Hao, C.-H. Chen, L.-J. Li, A. Singh, K. Tran, G. Clark, X. Xu, G. Berghuser, E. Malic, A. Knorr, and X. Li, Nat. Commun. 6, 8315 (2015).

${ }^{33}$ W. S. Edelstein and H. N. Spector, Surf. Sci. 224, 581-590 (1989).

${ }^{34}$ D. A. Kleinman, Phys. Rev. B 32, 3766-3771 (1985).

${ }^{35}$ P. Bigenwald, A. Kavokin, B. Gil, and P. Lefebvre, Phys. Rev. B 63, 035315 (2001).

${ }^{36}$ S. Schmitt-Rink, D. S. Chemla, and D. A. B. Miller, Phys. Rev. B 32, 6601-6609 (1985).

${ }^{37}$ A. Steinhoff, M. Rosner, F. Jahnke, T. Wehling, and C. Gies, Nano Lett. 14, 3743-3748 (2014).

${ }^{38}$ G. Moody, J. Schaibley, and X. Xu, J. Opt. Soc. Am. B 33, C39-C49 (2016).

${ }^{39}$ V. Huard, R. T. Cox, K. Saminadayar, A. Arnoult, and S. Tatarenko, Phys. Rev. Lett. 84, 187-190 (2000).

${ }^{40}$ C. Zhang, H. Wang, W. Chan, C. Manolatou, and F. Rana, Phys. Rev. B 89, 205436 (2014).

${ }^{41}$ G. Drake and Z.-C. Van, Chem. Phys. Lett. 229, 486-490 (1994).

${ }^{42}$ F. G. Pikus, Sov. Phys. Semicond. 26(1), 26-33 (1992).

${ }^{43}$ A. A. Klochikhin, V. P. Kochereshko, L. Besombes, G. Karczewski, T. Wojtowicz, and J. Kossut, Phys. Rev. B 83, 235329 (2011).

${ }^{44}$ A. Klochikhin, V. Kochereshko, and S. Tatarenko, J. Lumin. 154, 310-315 (2014).

${ }^{45}$ A. Ramasubramaniam, Phys. Rev. B 86, 115409 (2012).

${ }^{46}$ S. Latini, K. T. Winther, T. Olsen, and K. S. Thygesen, Nano Lett. 17, 938-945 (2017).

${ }^{47}$ M. M. Ugeda, A. J. Bradley, S.-F. Shi, F. H. da Jornada, Y. Zhang, D. Y. Qiu, W. Ruan, S.-K. Mo, Z. Hussain, Z.-X. Shen, F. Wang, S. G. Louie, and M. F. Crommie, Nat. Mater. 13, 1091-1095 (2014).

${ }^{48}$ N. Rytova, Vestn. Mosk. Univ. 3, 30 (1967).

${ }^{49}$ L. V. Keldysh, J. Exp. Theor. Phys. Lett. 29, 658 (1979).

${ }^{50}$ D. K. Efimkin and A. H. MacDonald, Phys. Rev. B 95, 035417 (2017).

${ }^{51}$ J. Jadczak, A. Delgado, L. Bryja, Y. Huang, and P. Hawrylak, Phys. Rev. B 95, 195427 (2017). 